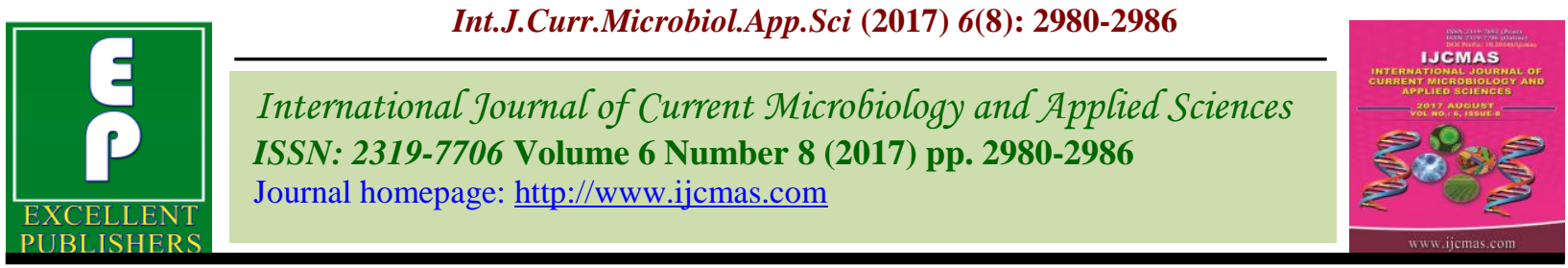

Original Research Article

https://doi.org/10.20546/ijcmas.2017.608.357

\title{
Efficacy of Entomopathogenic Fungi against Aphids on Okra
}

\author{
Ravi Palthiya* and R.V. Nakat \\ Department of Agricultural Entomology, M.P.K.V, Rahuri (413722), Maharashtra, India \\ *Corresponding author
}

\begin{tabular}{|c|c|}
\hline & A B S T R A C T \\
\hline $\begin{array}{l}\text { Beauveria } \\
\text { bassiana, } \\
\text { Metarhizium } \\
\text { anisopliae, } \\
\text { Verticillium } \\
\text { lecanii, Aphids, } \\
\text { Okra. }\end{array}$ & \multirow{3}{*}{$\begin{array}{l}\text { The field experiment was conducted during Kharif season of } 2013 \text { to study } \\
\text { the Efficacy of Entomopathogenic fungi against Aphids on Okra. During } \\
\text { the course of present investigation, three Entomopathogenic fungi were } \\
\text { tested for their effect at various combinations with each other at same } \\
\text { concentrations and compared with chemical insecticide dimethoate } 30 \mathrm{EC} \text {, } \\
\text { with a view to find out most effective treatment (s) on Aphids on Okra. The } \\
\text { experiment was conducted at P.G. Research Farm of Agril. Entomology } \\
\text { Department, Mahatma Phule Krishi Vidyapeeth, Rahuri. The influence of } \\
\text { different biopesticides and their combinations on aphids was studied during } \\
\text { the investigation. Thus, the results indicated that combination of } \\
\text { Entomopathogenic fungi as V. lecanii } 1.15 \% \mathrm{WP}+M \text {. anisopliae } 1.15 \% \\
\text { WP was the most effective treatment as compared to standard check } \\
\text { dimethoate for suppression of Aphids population on Okra. }\end{array}$} \\
\hline Article Info & \\
\hline & \\
\hline
\end{tabular}

\section{Introduction}

Okra (Bhendi) Abelmoschus esculentus (L.) Moench is one of the most important vegetable grown throughout the tropics and warmer parts of temperate zone. It is widely cultivated as a summer season crop in North India and Maharashtra. Okra is especially valued for its tender delicious fruits in different parts of country. Though it is mainly used as a fresh vegetable, it is also consumed as canned, dehydrated and frozen forms. Dry okra seeds contain 18 to 20 per cent oil, 20 to 23 per cent crude protein and good source of iodine (Barry et al., 1988). It has good export potential accounting for 60 per cent of fresh vegetable (Sharman and Arora, 1993). Though okra finds its origin in Central Africa, India stands top in area and production. It is cultivated in an area of 5.8 lakh hectares with an annual production 63.50 lakh tones with a productivity of $12.0 \mathrm{Mt} / \mathrm{ha}$ (Anonymous, 2013). In Maharashtra, okra cultivated in an area of 0.22 lakh hectares with an annual production 3.28 lakh tones/ha with a productivity of $14.90 \mathrm{Mt} / \mathrm{ha}$ (Ann, 2012-13). The major okra growing states include Andhra Pradesh, Uttar Pradesh, Bihar, Orissa, Karnataka, Maharashtra and Assam (Anonymous, 2013).

One of the most important constraints in production of okra is insect pests. As high as 72 species of insects have been recorded on crop (Srinivasa Rao and Rajendra, 2003) among which, the sucking pest complex 
consisting of aphids (Aphis gossypii Glover), leafhopper (Amrasca biguttula biguttula Ishida), whitefly (Bemisia tabacii. Gennadius) and Thrips (Thrips tabaci Lindeman) are major pest and causes 17.46 per cent yield loss in okra (Sarkar et al., 1996).

To tackle the pest menace, a number of chemical insecticides are liberally sprayed on this vegetable crop which leads to several problems like toxic residues, elimination of natural enemies, environmental disharmony and development of resistance. The demand is ever increasing for organically produced agricultural commodities all round the world and biological control agents have vital role to reduce the pest damage.

Okra being a fresh vegetable that is harvested at regular interval, it is critical to evaluate safer alternatives like botanicals and mycopathogens which possess no residual toxicity, is best suited for vegetables like okra, where we use fresh vegetables for consumption. Earlier workers tested bioefficacy of some of the indigenous materials against pests of okra (Jayakumar, 2002 and Dhanalakshmi, 2006) and reported their effect in reducing the pest population. Very meager information is available on the effect of Entomopathogenic fungi against okra Aphids. In this background, the present studies were carried out to evaluate the efficacy of Entomopathogenic fungi against okra Aphids.

\section{Materials and Methods}

The field trial was carried out at the experimental farm of Department of Agricultural Entomology, Post Graduate Institute, Mahatma Phule Krishi Vidhyapeeth, Rahuri, Dist. Ahmednagar, and Maharashtra during Kharif 2013-14 on variety of okra Phule Utkarsha in a randomized block design with three replications. Treatments of $B$. bassiana 1.15\% WP @ 5 gm/lit, $M$. anisopliae 1.15\% WP @ 5 gm/lit and $V$. lecanii 1.15\% WP @ 5 gm/lit and their combinations were tested in comparison with Dimethoate 30 EC $1.5 \mathrm{ml} /$ lit and untreated control (Table 1). Three sprays were imposed on need basis. Observations on Aphids was recorded one day before and 5, 10 and 15 days after spraying, on five randomly selected plants covering three leaves, one each from top, middle and bottom portion of the plant. The data were obtained and analysed statistically suggested by Panse and Sukhatme (1978).

\section{Results and Discussion}

The data on the efficacy of various biopesticides treatments on reducing Aphids population after first, second and third spraying are furnished in table 1, 2 and 3, respectively. The pretreatment counts were made a day before spraying indicated that there was no significant difference among the treatments.

At average of first spray indicated that, all the treatments were found superior in suppressing the aphid's population as compared to untreated control. The treatment dimethoate 30 EC was significantly superior over other treatment recorded 5.19 aphids/leaves/plant. Among biopesticides the treatment was combinations of $V$. lecanii $1.15 \% \mathrm{WP}+M$. anisopliae $1.15 \%$ WP spray in controlling aphids with survival population of 8.96 aphids/leaves/plant which were at par with the treatment $V$. lecanii $1.15 \% \mathrm{WP}, B$. bassiana $1.15 \% \mathrm{WP}+M$. anisopliae $1.15 \% \mathrm{WP}+V$. lecanii $1.15 \% \mathrm{WP}$ and $M$. anisopliae $1.15 \%$ WP recorded 9.68, 9.80 and 10.25aphids/leaves/plant respectively. It was followed by the treatments $V$. lecanii $1.15 \%$ $\mathrm{WP}+B$. bassiana $1.15 \%$ WP $1.15 \%$ WP (10.51aphids/leaves/plant), B. bassiana $1.15 \%$ $\mathrm{WP}+M$ anisopliae $1.15 \%$ WP (11.01aphids/leaves/plant) and B. bassiana $1.15 \%$ WP (14.61 aphids/leaves/plant), respectively (Table 1). 
Table.1 Efficacy of Entomopathogenic fungi against aphid on okra after first spray

\begin{tabular}{|c|c|c|c|c|c|c|c|}
\hline \multirow{3}{*}{ Tr. No. } & \multirow{3}{*}{ Treatments } & \multicolumn{6}{|c|}{ Number of aphids/leaves/plant } \\
\hline & & \multirow{2}{*}{$\begin{array}{l}\text { Dosage } \\
\text { Qty/ lit. }\end{array}$} & \multicolumn{5}{|c|}{ I Spray } \\
\hline & & & DBS & 5 DAS & 10 DAS & 15 DAS & Average \\
\hline $\mathrm{T}_{1}$ & B. bassiana $1.15 \% \mathrm{WP}$ & $5 \mathrm{gm} / \mathrm{lit}$ & $\begin{array}{l}18.09 \\
(4.3) \\
\end{array}$ & $\begin{array}{l}14.12 \\
(3.86) \\
\end{array}$ & $\begin{array}{l}13.10 \\
(3.68)\end{array}$ & $\begin{array}{c}16.4 \\
(4.10)\end{array}$ & $\begin{array}{l}14.61 \\
(3.89) \\
\end{array}$ \\
\hline $\mathrm{T}_{2}$ & M. anisopliae $1.15 \% \mathrm{WP}$ & $5 \mathrm{gm} / \mathrm{lit}$ & $\begin{array}{l}17.57 \\
(4.24)\end{array}$ & $\begin{array}{l}11.91 \\
(3.52)\end{array}$ & $\begin{array}{c}8.77 \\
(3.04)\end{array}$ & $\begin{array}{l}10.07 \\
(3.25)\end{array}$ & $\begin{array}{l}10.25 \\
(3.28)\end{array}$ \\
\hline $\mathrm{T}_{3}$ & V. lecanii $1.15 \% \mathrm{WP}$ & $5 \mathrm{gm} / \mathrm{lit}$ & $\begin{array}{l}18.56 \\
(4.32)\end{array}$ & $\begin{array}{l}11.68 \\
(3.49)\end{array}$ & $\begin{array}{c}8.71 \\
(3.03)\end{array}$ & $\begin{array}{c}9.08 \\
(3.09)\end{array}$ & $\begin{array}{c}9.68 \\
(3.18)\end{array}$ \\
\hline $\mathrm{T}_{4}$ & V. lecanii $1.15 \% \mathrm{WP}+\mathrm{M}$. anisopliae $1.15 \% \mathrm{WP}$ & 5 gm/lit. each & $\begin{array}{l}18.59 \\
(4.36) \\
\end{array}$ & $\begin{array}{l}11.26 \\
(3.37) \\
\end{array}$ & $\begin{array}{c}7.44 \\
(2.82) \\
\end{array}$ & $\begin{array}{c}7.77 \\
(2.86) \\
\end{array}$ & $\begin{array}{c}8.96 \\
(3.06) \\
\end{array}$ \\
\hline $\mathrm{T}_{5}$ & B. bassiana $1.15 \% \mathrm{WP}+\mathrm{M}$. anisopliae $1.15 \% \mathrm{WP}$ & 5 gm/lit. each & $\begin{array}{l}17.51 \\
(4.24)\end{array}$ & $\begin{array}{l}12.57 \\
(3.61)\end{array}$ & $\begin{array}{l}10.20 \\
(3.26)\end{array}$ & $\begin{array}{l}10.95 \\
(3.38)\end{array}$ & $\begin{array}{l}11.01 \\
(3.39)\end{array}$ \\
\hline $\mathrm{T}_{6}$ & V. lecanii $1.15 \% \mathrm{WP}+$ B. bassiana $1.15 \% \mathrm{WP} 1.15 \% \mathrm{WP}$ & $5 \mathrm{gm} /$ lit. each & $\begin{array}{l}19.32 \\
(4.43) \\
\end{array}$ & $\begin{array}{l}12.05 \\
(3.54) \\
\end{array}$ & $\begin{array}{c}9.71 \\
(3.19)\end{array}$ & $\begin{array}{l}10.66 \\
(3.34) \\
\end{array}$ & $\begin{array}{l}10.51 \\
(3.32) \\
\end{array}$ \\
\hline $\mathrm{T}_{7}$ & $\begin{array}{l}\text { B. bassiana } 1.15 \% \mathrm{WP}+\mathrm{M} \text {. anisopliae } 1.15 \% \mathrm{WP}+\mathrm{V} \text {. } \\
\text { lecanii } 1.15 \% \mathrm{WP}\end{array}$ & 5 gm/lit. each & $\begin{array}{l}18.20 \\
(4.29)\end{array}$ & $\begin{array}{l}11.48 \\
(3.46)\end{array}$ & $\begin{array}{c}8.52 \\
(3.00)\end{array}$ & $\begin{array}{c}9.41 \\
(3.14)\end{array}$ & $\begin{array}{c}9.80 \\
(3.21)\end{array}$ \\
\hline $\mathrm{T}_{8}$ & Dimethoate 30EC & $1.5 \mathrm{ml} / \mathrm{lit}$ & $\begin{array}{l}19.25 \\
(4.44)\end{array}$ & $\begin{array}{c}6.13 \\
(2.57)\end{array}$ & $\begin{array}{c}3.54 \\
(2.01)\end{array}$ & $\begin{array}{c}5.88 \\
(2.53)\end{array}$ & $\begin{array}{l}5.19 \\
(2.38)\end{array}$ \\
\hline $\mathrm{T}_{9}$ & Untreated control & - & $\begin{array}{l}19.48 \\
(4.45)\end{array}$ & $\begin{array}{l}23.12 \\
(4.86)\end{array}$ & $\begin{array}{l}27.15 \\
(5.26)\end{array}$ & $\begin{array}{c}27.9 \\
(5.33)\end{array}$ & $\begin{array}{l}26.06 \\
(5.15)\end{array}$ \\
\hline & $\mathrm{SE} \pm$ & - & 0.23 & 0.17 & 0.10 & 0.12 & 0.08 \\
\hline & CD at $5 \%$ & - & NS & 0.51 & 0.30 & 0.36 & 0.24 \\
\hline & $\mathrm{CV} \%$ & - & 9.46 & 8.26 & 9.81 & 10.14 & 8.16 \\
\hline
\end{tabular}

Figures in the parentheses are $(\sqrt{x+0.5})$ transformations, DBS-Day before spraying \& DAS-Days after spraying 
Table.2 Efficacy of Entomopathogenic fungi against aphid on okra after Second spray

\begin{tabular}{|c|c|c|c|c|c|c|}
\hline \multirow{3}{*}{ Tr. No. } & \multirow{3}{*}{ Treatments } & \multicolumn{5}{|c|}{ Number of aphids/leaves/plant } \\
\hline & & \multirow{2}{*}{$\begin{array}{l}\text { Dosage } \\
\text { Qty/ lit. }\end{array}$} & \multicolumn{4}{|c|}{ II Spray } \\
\hline & & & 5 DAS & 10 DAS & 15 DAS & Average \\
\hline $\mathrm{T}_{1}$ & B. bassiana $1.15 \% \mathrm{WP}$ & $5 \mathrm{gm} / \mathrm{lit}$ & $\begin{array}{l}12.81 \\
(3.64)\end{array}$ & $\begin{array}{l}14.26 \\
(3.84)\end{array}$ & $\begin{array}{l}13.05 \\
(3.68)\end{array}$ & $\begin{array}{l}13.37 \\
(3.72)\end{array}$ \\
\hline $\mathrm{T}_{2}$ & M. anisopliae $1.15 \% \mathrm{WP}$ & $5 \mathrm{gm} / \mathrm{lit}$ & $\begin{array}{c}9.20 \\
(3.11) \\
\end{array}$ & $\begin{array}{c}8.91 \\
(3.06) \\
\end{array}$ & $\begin{array}{c}8.99 \\
(3.07) \\
\end{array}$ & $\begin{array}{c}9.03 \\
(3.09) \\
\end{array}$ \\
\hline $\mathrm{T}_{3}$ & V. lecanii $1.15 \% \mathrm{WP}$ & $5 \mathrm{gm} / \mathrm{lit}$ & $\begin{array}{c}8.29 \\
(2.96)\end{array}$ & $\begin{array}{c}7.51 \\
(2.82) \\
\end{array}$ & $\begin{array}{c}8.66 \\
(3.03) \\
\end{array}$ & $\begin{array}{c}7.78 \\
(2.88)\end{array}$ \\
\hline $\mathrm{T}_{4}$ & V. lecanii $1.15 \% \mathrm{WP}+\mathrm{M}$. anisopliae $1.15 \% \mathrm{WP}$ & $5 \mathrm{gm} /$ lit. each & $\begin{array}{c}7.18 \\
(2.77) \\
\end{array}$ & $\begin{array}{c}6.95 \\
(2.72) \\
\end{array}$ & $\begin{array}{c}7.84 \\
(2.88) \\
\end{array}$ & $\begin{array}{c}7.70 \\
(2.86) \\
\end{array}$ \\
\hline $\mathrm{T}_{5}$ & B. bassiana $1.15 \% \mathrm{WP}+\mathrm{M}$. anisopliae $1.15 \% \mathrm{WP}$ & $5 \mathrm{gm} /$ lit. each & $\begin{array}{l}10.51 \\
(3.31)\end{array}$ & $\begin{array}{c}9.26 \\
(3.12)\end{array}$ & $\begin{array}{l}12.49 \\
(3.60)\end{array}$ & $\begin{array}{l}10.75 \\
(3.35)\end{array}$ \\
\hline $\mathrm{T}_{6}$ & V. lecanii $1.15 \% \mathrm{WP}+$ B. bassiana $1.15 \% \mathrm{WP} 1.15 \% \mathrm{WP}$ & $5 \mathrm{gm} / \mathrm{lit}$. each & $\begin{array}{l}10.03 \\
(3.24) \\
\end{array}$ & $\begin{array}{c}9.96 \\
(3.23) \\
\end{array}$ & $\begin{array}{c}9.44 \\
(3.14)\end{array}$ & $\begin{array}{c}9.81 \\
(3.21)\end{array}$ \\
\hline $\mathrm{T}_{7}$ & $\begin{array}{l}\text { B. bassiana } 1.15 \% \mathrm{WP}+\mathrm{M} . \text { anisopliae } 1.15 \% \mathrm{WP}+\mathrm{V} . \\
\text { lecanii } 1.15 \% \mathrm{WP}\end{array}$ & $5 \mathrm{gm} / \mathrm{lit}$. each & $\begin{array}{c}8.63 \\
(3.01) \\
\end{array}$ & $\begin{array}{c}8.84 \\
(3.05) \\
\end{array}$ & $\begin{array}{c}9.33 \\
(3.13) \\
\end{array}$ & $\begin{array}{c}8.93 \\
(3.07) \\
\end{array}$ \\
\hline $\mathrm{T}_{8}$ & Dimethoate 30EC & $1.5 \mathrm{ml} / \mathrm{lit}$ & $\begin{array}{c}4.64 \\
(2.26)\end{array}$ & $\begin{array}{c}5.74 \\
(2.49)\end{array}$ & $\begin{array}{c}7.54 \\
(2.83)\end{array}$ & $\begin{array}{c}5.98 \\
(2.54)\end{array}$ \\
\hline $\mathrm{T}_{9}$ & Untreated control & - & $\begin{array}{l}24.64 \\
(5.01)\end{array}$ & $\begin{array}{l}23.33 \\
(4.88)\end{array}$ & $\begin{array}{l}26.18 \\
(5.16)\end{array}$ & $\begin{array}{l}24.72 \\
(5.02)\end{array}$ \\
\hline & $\mathbf{S E} \pm$ & - & 0.12 & 0.11 & 0.08 & 0.06 \\
\hline & CD at $5 \%$ & - & 0.37 & 0.33 & 0.23 & 0.17 \\
\hline & CV \% & - & 9.17 & 10.71 & 11.16 & 8.05 \\
\hline
\end{tabular}

Figures in the parentheses are $(\sqrt{x+0.5})$ transformations, DBS-Day before spraying \& DAS-Days after spraying 
Table.3 Efficacy of Entomopathogenic fungi against aphid on okra after Third spray

\begin{tabular}{|c|c|c|c|c|c|c|c|}
\hline \multirow{3}{*}{ Tr. No. } & \multirow{3}{*}{ Treatments } & \multicolumn{5}{|c|}{ Number of aphids/leaves/plant } & \multirow{3}{*}{$\begin{array}{c}\text { Average } \\
\text { of three } \\
\text { sprays }\end{array}$} \\
\hline & & \multirow{2}{*}{$\begin{array}{l}\text { Dosage } \\
\text { Qty/ lit. }\end{array}$} & \multicolumn{4}{|c|}{ III Spray } & \\
\hline & & & 5 DAS & 10 DAS & 15 DAS & Average & \\
\hline $\mathrm{T}_{1}$ & B. bassiana $1.15 \% \mathrm{WP}$ & $5 \mathrm{gm} / \mathrm{lit}$ & $\begin{array}{l}11.33 \\
(3.44)\end{array}$ & $\begin{array}{l}10.16 \\
(3.26)\end{array}$ & $\begin{array}{c}8.36 \\
(2.96)\end{array}$ & $\begin{array}{c}9.95 \\
(3.23)\end{array}$ & $\begin{array}{l}11.06 \\
(3.40)\end{array}$ \\
\hline $\mathrm{T}_{2}$ & M. anisopliae $1.15 \% \mathrm{WP}$ & $5 \mathrm{gm} / \mathrm{lit}$ & $\begin{array}{c}7.46 \\
(2.82)\end{array}$ & $\begin{array}{c}7.00 \\
(2.74)\end{array}$ & $\begin{array}{c}7.39 \\
(2.81)\end{array}$ & $\begin{array}{c}7.28 \\
(2.79)\end{array}$ & $\begin{array}{c}7.87 \\
(2.89)\end{array}$ \\
\hline $\mathrm{T}_{3}$ & V. lecanii $1.15 \% \mathrm{WP}$ & $5 \mathrm{gm} / \mathrm{lit}$ & $\begin{array}{c}6.44 \\
(2.63)\end{array}$ & $\begin{array}{c}5.96 \\
(2.54)\end{array}$ & $\begin{array}{c}6.04 \\
(2.54)\end{array}$ & $\begin{array}{c}6.15 \\
(2.57)\end{array}$ & $\begin{array}{c}7.02 \\
(2.74)\end{array}$ \\
\hline $\mathrm{T}_{4}$ & V. lecanii $1.15 \% \mathrm{WP}+\mathrm{M}$. anisopliae $1.15 \% \mathrm{WP}$ & $5 \mathrm{gm} /$ lit. each & $\begin{array}{c}6.40 \\
(2.62)\end{array}$ & $\begin{array}{c}5.14 \\
(2.37)\end{array}$ & $\begin{array}{c}5.84 \\
(2.52)\end{array}$ & $\begin{array}{c}5.80 \\
(2.51)\end{array}$ & $\begin{array}{c}6.73 \\
(2.69)\end{array}$ \\
\hline $\mathrm{T}_{5}$ & B. bassiana $1.15 \% \mathrm{WP}+\mathrm{M}$. anisopliae $1.15 \% \mathrm{WP}$ & $5 \mathrm{gm} / \mathrm{lit}$. each & $\begin{array}{l}10.65 \\
(3.34)\end{array}$ & $\begin{array}{c}8.85 \\
(3.06)\end{array}$ & $\begin{array}{c}8.24 \\
(2.95)\end{array}$ & $\begin{array}{c}9.25 \\
(3.12)\end{array}$ & $\begin{array}{c}9.31 \\
(3.13)\end{array}$ \\
\hline $\mathrm{T}_{6}$ & V. lecanii $1.15 \% \mathrm{WP}+$ B. bassiana1.15\% WP $1.15 \% \mathrm{WP}$ & $5 \mathrm{gm} / \mathrm{lit}$. each & $\begin{array}{c}7.59 \\
(2.84)\end{array}$ & $\begin{array}{c}7.36 \\
(2.80)\end{array}$ & $\begin{array}{c}7.72 \\
(2.87)\end{array}$ & $\begin{array}{c}7.56 \\
(2.84)\end{array}$ & $\begin{array}{c}8.18 \\
(2.95)\end{array}$ \\
\hline $\mathrm{T}_{7}$ & $\begin{array}{l}\text { B. bassiana } 1.15 \% \mathrm{WP}+\mathrm{M} \text {. anisopliae } 1.15 \% \mathrm{WP}+\mathrm{V} \text {. } \\
\text { lecanii } 1.15 \% \mathrm{WP}\end{array}$ & $5 \mathrm{gm} / \mathrm{lit}$. each & $\begin{array}{c}7.15 \\
(2.76)\end{array}$ & $\begin{array}{c}6.60 \\
(2.64)\end{array}$ & $\begin{array}{c}6.48 \\
(2.64)\end{array}$ & $\begin{array}{c}6.74 \\
(2.69)\end{array}$ & $\begin{array}{c}7.51 \\
(2.82)\end{array}$ \\
\hline $\mathrm{T}_{8}$ & Dimethoate 30EC & $1.5 \mathrm{ml} / \mathrm{lit}$ & $\begin{array}{c}1.96 \\
(1.56)\end{array}$ & $\begin{array}{c}0.98 \\
(1.21)\end{array}$ & $\begin{array}{c}1.82 \\
(1.52)\end{array}$ & $\begin{array}{c}1.59 \\
(1.44)\end{array}$ & $\begin{array}{c}3.61 \\
(2.03)\end{array}$ \\
\hline $\mathrm{T}_{9}$ & Untreated control & - & $\begin{array}{l}24.28 \\
(4.98)\end{array}$ & $\begin{array}{l}20.50 \\
(4.58)\end{array}$ & $\begin{array}{l}20.97 \\
(4.63)\end{array}$ & $\begin{array}{l}21.92 \\
(4.73)\end{array}$ & $\begin{array}{l}21.64 \\
(4.71)\end{array}$ \\
\hline & $\mathrm{SE} \pm$ & - & 0.08 & 0.09 & 0.07 & 0.06 & 0.06 \\
\hline & CD at $5 \%$ & - & 0.24 & 0.27 & 0.32 & 0.18 & 0.18 \\
\hline & $\mathrm{CV} \%$ & - & 13.96 & 14.98 & 10.50 & 7.73 & 7.42 \\
\hline
\end{tabular}

Figures in the parentheses are $(\sqrt{x+0.5})$ transformations, DBS-Day before spraying \& DAS-Days after spraying 
At average second spray indicated that, all the treatments were found superior in suppressing the aphids' population as compared to untreated control. The treatment dimethoate 30 EC was significantly superior over other treatment recorded 4.64 aphids/leaves/plant. The next promosing treatment was combination of $V$. lecanii $1.15 \% \mathrm{WP}+M$. anisopliae $1.15 \% \mathrm{WP}$ spray in controlling aphids with survival population 7.70 aphids/leaves/plant which were at par with the treatments $V$. lecanii $1.15 \%$ WP 7.78 aphids/leaves/plant and. It was followed by the treatment of $B$. bassiana $1.15 \% \mathrm{WP}+M$. anisopliae $1.15 \% \mathrm{WP}+V$. lecanii $1.15 \% \mathrm{WP}$ recorded (8.93aphids/leaves/plant). The performing treatments in order to their merit were $M$. anisopliae $1.15 \% \mathrm{WP}, V$. lecanii $1.15 \% \mathrm{WP}+B$. bassiana $1.15 \% \mathrm{WP}, B$. bassiana $1.15 \% \mathrm{WP}+M$. anisopliae $1.15 \%$ WP and B. bassiana $1.15 \%$ were recorded of survival population in the range of 9.03 to 13.37 aphids/leaves/plant, respectively (Table2).

At average of third spray after treatments, the mean aphids' population after third spray revealed that the least aphids count was recorded in standard check dimethoate $30 \mathrm{EC}$ treatment (1.59aphids/leaves/plant) was statistically found superior over other treatments. The next best treatment was combination of $V$. lecanii $1.15 \% \mathrm{WP}+M$. anisopliae $1.15 \% \mathrm{WP}$ in controlling aphids with survival population of (5.80 aphids/leaves/plant), which were at par with the treatments $V$. lecanii $1.15 \% \mathrm{WP}$ and $B$. bassiana $1.15 \% \mathrm{WP}+M$. anisopliae $1.15 \%$ $\mathrm{WP}+V$. lecanii $1.15 \% \mathrm{WP}$ recorded 6.15 and 6.74 aphids/leaves/plant. It was followed by the treatments $M$. anisopliae $1.15 \%$ WP (7.28 aphids/leaves/plant), V. lecanii $1.15 \% \mathrm{WP}+$ B. bassiana $1.15 \%$ WP $\quad(7.56$ aphids/leaves/plant) and B. bassiana $1.15 \%$ $\mathrm{WP}+M$. anisopliae $1.15 \%$ WP $(9.25$ aphids/leaves/plant). The least significant treatment was $B$. bassiana $1.15 \% \mathrm{WP}$ was recorded 9.95 aphids/leaves/plant respectively (Table 3).

The present findings are in agreement with Dromph et al., (1996) who studied the pathogenicity of $V$. lecanii and $B$. bassiana against cereal aphid, Rhopalosiphum padi and Metalophium dirhodium and found that $V$. lecanii was more effective than B. bassiana.

Safavi et al., (2002) showed that V. lecanii significantly increased aphid mortality due to mycosis from 45.55 per at dose of $10^{4}$ conidia/ml to 95.55 per cent at $10^{8}$ conidia $/ \mathrm{ml}$.

Neelam et al., (2003) tested the L. lecanii at

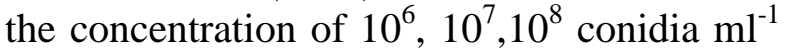
against $L$. erysimi and reported the highest mortality of 80 per cent $96 \mathrm{hrs}$ of treatment at the concentration of $10^{8}$ conidia $\mathrm{ml}^{-1}$. The results obtained in could support the earlier finding of Grunberg et al., (1988), karinadah et al., (1996).

Efficacy of mycopathogen against aphids is in accordance with Nirmala et al., (2006), according to whom the $\mathrm{V} 11$ isolate of $\mathrm{V}$. lecanii recorded maximum mortality of $\mathrm{A}$. Craccivora and A. gossypii. Further, more efficacy of M.anisopliae against aphid was in accordance with Masuda and Kikuchi. (1992), Ekeshi et al., (2000) and B.basiana was found effective in controlling the aphids supported by Liu-yin-Quan et al., (1999) and Zang et al., (2001).

The combination of $V$. lecanii $1.15 \% \mathrm{WP}+$ M. ansiopliae $1.15 \% \mathrm{WP}$ was found to be the most effective treatment for suppression of Aphids population of $\mathrm{O}$

\section{References}

Anonymous, 2012. Nat, Hort, Board, Indi, Hort. Database 2012. 
Anonymous, 2013. Nat, Hort. Board, Indi. Hort. Database 2013.

Bary, S, K., Kalra, C.L., Shegal, R.c., Kulkarni, S.G., Sukhvirkaur, Arora, S.K. and Sharma, B.R., 1988. Quality Charcteristics of seeds of five okra (Abelmoschus esculentus L.) cultivars, J.Food Sci. and Technol., 25:303 305.

Ekesi, Akpa, A.D., Onu, I. and ogunlana, M.O.2000. Entomopathogenicity of Beauvereia bassian and Metarrhizium anisopliae to the cowpea aphid, Aphis craccivora Koch (Homoptera; Aphididae). Arc. Of Phytopath. And P1.Prot, 33(2):171-180.

Grunberg, M., Adam, H., Walter, C. and Hirte, W.F. 1988 Possibilites for using the entomopathogenci fungus Verticillium licanii (Zimm.) Viegasfor biological control of aphids in crops under glass and plastic. J. of Nachrichlenblast Furden Pflamzenschutz -in - DDR, 42(9).

Jaya Kumar P., 2006. Bioefficacy of botanicals and biogens on sucking pests of cotton. Ann.Pl.Protec, Sci., 14(1):46:103-112.
Karindah, 1996. Virulence of the fungus, V.lecanii Zimm. Against Aphis gossypii Glover. Agrivita, 19(1).

Neelam, J., Brar, K.S., Maninder, S., Joshi, N. and Shenhmar, M. 2003. Preliminary evaluation of $V$. lecanji (Zimm.) viegas against Lipaphis erysimi (Kalt.). Insect Envi, 9(3):

Nirmala et al., 2006. Effect of entomopathogens on mortality of three aphid species. J. Biological Control, 20 (1):89-94.

Panse V.G., and Sukhatme P.V., 1978. Statistical methods for Agricultural Works, Indian Council of Agricultural Research, New Delhi.

Safavi, S. A., Rassulian, G. R., Askary. 2002, Pathogenicity and virulence of entomopathogenous fungus, $V$. lecanii against (Zimm.) Viegas on the pea aphid, Acrythosiphon pisum (Harris). J.sci. and Tech. Agri. And Natural Resources, 6(1): 245-255.

Zang, et al., 2001. Biological characteristics of Beauveria bassiana and its virulence against wheat aphid, Myzus Persicae. J. South West Agril. Univ., 23(2)

\section{How to cite this article:}

Ravi Palthiya and Nakat, R.V. 2017. Efficacy of Entomopathogenic fungi against Aphids on Okra. Int.J.Curr.Microbiol.App.Sci. 6(8): 2980-2986.

doi: https://doi.org/10.20546/ijcmas.2017.608.357 